\title{
Regional functional synchronizations in dementia with Lewy bodies and Alzheimer's disease
}

\author{
Luis R. Peraza, ${ }^{1,2}$ Sean J. Colloby, ${ }^{1}$ Liam Deboys, ${ }^{1}$ John T. O'Brien, ${ }^{3}$ Marcus Kaiser ${ }^{4}$ \\ and John-Paul Taylor ${ }^{1}$ \\ ${ }^{1}$ Institute of Neuroscience, Campus for Ageing and Vitality, Newcastle University, Newcastle upon Tyne, NE4 5PL, UK \\ ${ }^{2}$ Newcastle University Institute of Ageing, Campus for Ageing and Vitality, Newcastle University, Newcastle upon Tyne, NE4 5PL, UK \\ ${ }^{3}$ Department of Psychiatry, Cambridge Biomedical Campus, University of Cambridge School of Clinical Medicine, Cambridge, CB2 OSZ, UK \\ ${ }^{4}$ Interdisciplinary Computing and Complex BioSystems (ICOS) research group, School of Computing Science, Newcastle University, Newcastle upon Tyne, \\ NE1 7RU, UK
}

Background: Dementia with Lewy bodies (DLB) is a common cause of dementia in the elderly population after Alzheimer's disease (AD), and at early stages differential diagnosis between DLB and AD might be difficult due to their symptomatic overlap, e.g. cognitive and memory impairments. We aimed to investigate functional brain differences between both diseases in patients recently diagnosed.

Methods: We investigated regional functional synchronizations using regional homogeneity (ReHo) in patients clinically diagnosed with $\mathrm{DLB}(n=19)$ and $\mathrm{AD}(n=18)$, and for comparisons we also included healthy controls (HC, $n=16)$. Patient groups were matched by age, education, and by the level of cognitive impairment (MMSE $p$-value $=0.36)$. Additionally, correlations between ReHo values and clinical scores were investigated.

Results: The DLB group showed lower ReHo in sensory-motor cortices and higher ReHo in left middle temporal gyrus when compared with HCs ( $p$-value $<0.001$ uncorrected). The AD group demonstrated lower $\mathrm{ReHo}$ in the cerebellum and higher ReHo in the left/right lingual gyri, precuneus cortex, and other occipital and parietal regions ( $p$-value $<0.001$ uncorrected).

Conclusions: Our results agree with previous ReHo investigations in Parkinson's disease (PD), suggesting that functional alterations in motor-related regions might be a characteristic of the Lewy body disease spectrum. However, our results in $\mathrm{AD}$ contradict previously reported findings for this disease and ReHo, which we speculate are a reflection of compensatory brain responses at early disease stages. ReHo differences between patient groups were at regions related to the default mode and sensory-motor resting state networks which might reflect the aetiological divergences in the underlying disease processes between $\mathrm{AD}$ and DLB.

Key words: Dementia with Lewy bodies (DLB), Alzheimer's disease (AD), magnetic resonance imaging (MRI), hallucinations, neuroimaging

\section{Introduction}

DLB is a common cause of dementia, accounting for $4 \%-8 \%$ of dementia cases (McKeith et al., 2007), and it is characterized by three core clinical symptoms: cognitive fluctuations, visual hallucinations, and Parkinsonism (McKeith et al., 2005). There is now a growing body of neuroimaging research on DLB where this dementia is compared with $\mathrm{AD}$ in order to improve differential diagnosis between these two conditions,

Correspondence should be addressed to: Luis R. Peraza, Institute of Neuroscience, Campus for Ageing and Vitality, Newcastle University, Newcastle upon Tyne, NE4 5PL, UK. Phone: 0191208 1125. Email: luis.peraza-rodriguez@newcastle.ac.uk. Received 16 Nov 2015; revision requested 3 Jan 2016; revised version received 21 Jan 2016; accepted 7 Feb 2016. First published online 15 March 2016. as well as to further our understanding of their neurobiological divergences (Mukaetova-Ladinska, 2015).

In the current investigation, we were interested in studying resting state functional magnetic resonance imaging (rs-fMRI) alterations in DLB using an alternative approach of ReHo (Zang et al., 2004). ReHo evaluates local connectivity in rs-fMRI under the assumption that the brain's spontaneous functional synchronizations occur in clusters between neuronal groups and their nearest neighbors. In this regard, the average ReHo activity of $\mathrm{HCs}$ resembles the default mode network (DMN) (Wu et al., 2007), with higher ReHo in the frontal and precuneal cortices. The DMN is the most studied of the resting state networks and it has been posited that it relates to mind 
wandering and the recalling of episodic memory (Andrews-Hanna et al., 2010). Previous investigations have found abnormal DMN in AD (Agosta et al., 2012), which concurs with the reported decreased ReHo in the precuneus, posterior cingulate, and temporal cortices in $\mathrm{AD}$ ( $\mathrm{He}$ et al., 2007; Zhang et al., 2012).

In DLB, symptomatic overlap exists with $\mathrm{AD}$ which may be driven by the concurrent presence of amyloid pathology in this disease (Donaghy et al., 2015). However, we hypothesized that ReHo would have utility in identifying alterations in local connectivity in DLB not only confined to regions associated with amyloid deposition (e.g. the DMN), but also in regions which have been previously associated with the core clinical symptoms which typify DLB such as cognitive fluctuations, visual hallucinations, and Parkinsonism.

\section{Methods}

\section{Participants and assessment}

The study comprised 63 participants; 22 were diagnosed as DLB, 24 as $\mathrm{AD}$, and $17 \mathrm{HCs}$. Patients were recruited from members of the local population who had been referred to local old age psychiatry and neurology services. Diagnosis of patient groups was carried out independently by two experienced clinicians. Participants with $\mathrm{AD}$ fulfilled the revised criteria of the National Institute of Neurological and Communicative Diseases and Stroke/AD and Related Disorders Association for probable AD. DLB patients met the criteria for probable DLB, including the presence of two out of three core symptoms: cognitive fluctuations, visual hallucinations, and Parkinsonism (McKeith et al., 2005). Nine out of the 22 DLB patients had dopaminergic imaging, all of whom showed abnormal bilateral uptake. Clinical assessment included the MiniMental State Examination (MMSE), Cambridge Cognitive Examination (CAMCOG), and Unified Parkinson's disease rating scale (UPDRS). In order to assess the level of complex visual hallucinations, the hallucinations subscale questionnaire of the neuropsychiatric inventory test (NPI ${ }^{\text {hall }}$ ) was completed by the patients' caregivers. At the time of MRI scanning, the majority of patients were taking acetylcholinesterase inhibitors (AChEI; 21 DLBs and $24 \mathrm{ADs}$ ) and ten DLB patients were on Levodopa treatment. The majority of the control group had no history of psychiatric condition (one control participant was on antidepressants), all showed no evidences of neurological brain disease and got MMSE score of $>27$. All participants' structural MRIs were reviewed to discard other conditions or sources of dementia, such as vascular lesions/infarcts. None of the participants discontinued their medications during the study. Approval for this investigation was granted by the Newcastle Ethics Committee and all participants gave informed consent.

\section{MRI and fMRI pre-processing}

Structural and functional acquisition parameters for this cohort of participants have been reported elsewhere (Peraza et al., 2015). For this investigation, fMRIs were first pre-processed using Statistical Parametric Mapping software (SPM12, http://www.fil.ion.ucl.ac.uk/spm/). The steps included slice timing correction with posterior volume realignment to correct for movement. Then, structural MRIs were co-registered to the mean fMRI image from the realignment step and normalized to MNI space with a $2 \times 2 \times 4 \mathrm{~mm}$ voxel resolution. Parameters from the previous normalization were used to normalize the fMRI images to MNI space with a $2 \times 2 \times 4 \mathrm{~mm}$ voxel resolution.

Motion time series (translations and rotations) were analyzed for motion exclusion criteria. Participants who showed a deviation of $>2 \mathrm{~mm}$ or $>2^{\circ}$ from the reference image (first volume) were excluded. To further assure data quality, the six motion parameters from the realignment step were regressed from all participants' fMRIs.

\section{Regional homogeneity}

ReHo images were computed using the REST toolbox (Song et al., 2011). Briefly, ReHo is estimated using the Kendall's coefficient of concordance (KCC) between a voxel and its nearest neighbors:

$$
K C C=\frac{\sum\left(R_{i}\right)^{2}-n(\bar{R})^{2}}{\frac{1}{12} K^{2}\left(n^{3}-n\right)}
$$

Where $R_{i}$ is the sum rank of the $i$ th time point; $\bar{R}=((n+1) K) / 2$ is the mean of the $R_{i} \mathrm{~s} ; K$ is the number of time series within a measured cluster (27 in this analysis); and $n$ is the number of ranks ( $n=128$ in the present analysis). Before ReHo image estimation, the fMRIs were detrended and bandpass filtered $(0.01-0.08 \mathrm{~Hz})$. As final step, the ReHo images were normalized by the average $\mathrm{ReHo}$ value and spatially smoothed with a $10-\mathrm{mm}$ full width half maximum spatial (FWHM) filter.

\section{Functional and anatomical ReHo evaluations}

We estimated average ReHo values from functional and anatomical seeds. Functional seeds were 
taken from significant clusters arising from ReHo comparisons between the patient groups and the HC group. Anatomical seeds were defined by the Anatomical Automatic Labeling (AAL) atlas available in the MarsBar SPM toolbox (Brett et al., 2002). The regions assessed were chosen according to their relevance in previous $\mathrm{ReHo}$ investigations in $\mathrm{AD}$ and PD. These were the cuneus and precuneus (He et al., 2007), sensory-motor cortices (pre-/postcentral gyri and supplementary motor areas) (Choe et al., 2013), and thalamus and putamen (Wu et al., 2009a). Marsbar SPM toolbox was also used to extract the average ReHo from the functional and anatomical seeds. Correlations between the average ReHo values and clinical scores - MMSE, CAMCOG, UPDRS, CAF, and NPI ${ }^{\text {hall }}$ - were also examined.

\section{Voxel-based morphometry}

In order to assess grey matter differences in our participant groups, we carried out an independent voxel-based morphometry (VBM) analysis using the DARTEL registration algorithm in SPM12. DARTEL maps were posteriorly smoothed with an 8-mm FWHM filter.

\section{Statistical analysis}

Analysis of the demographic and clinical variables was performed using SPSS (version 21, IBM). Statistical comparisons of the motion parameters (translations and rotations) for the three groups were assessed by Kruskal-Wallis tests using the motion/rotation formula as the test statistic (Peraza et al., 2015); motion/rotation $=(M-1)^{-1} \sum_{i=2}^{M} \sqrt{\left|x_{i}-x_{i-1}\right|^{2}+\left|y_{i}-y_{i-1}\right|^{2}+\left|z_{i}-z_{i-1}\right|^{2}}$, where $x, y$, and $z$ are the three motion/rotation parameters and $M$ is the length of the fMRI $(M=128$ in the present study).

Comparisons between groups for ReHo were implemented with non-parametric permutations $(10,000$ permutations, two-sample $t$-test) using the non-parametric SPM toolbox (SnPM, http://warwick.ac.uk/snpm), with age and gender as covariates of no interest. Results were considered to be significant at a voxel $p$-value of $<0.001$ uncorrected, which also defined the functional seeds to study correlations with clinical variables.

Average ReHo values from the functional and anatomical seeds were assessed for correlations with clinical variables using Spearman's rank correlation (SPSS) and group comparisons using the average ReHo values from anatomical seeds were assessed with two-tailed unpaired $t$-tests after regressing out age and gender (Matlab).

Grey matter volume differences were firstly assessed by an ANCOVA, followed by post hoc unpaired two-sample $t$-tests to find differences between the groups. For all VBM analyses, age, sex, and total intracranial volume (TIV) were included as nuisance covariates.

\section{Results}

From the 63 participants in our study, one HC, 6 ADs, 3 DLBs were excluded from our analysis due to excessive motion ( $>2 \mathrm{~mm}$ translations), leaving a final study cohort of $16 \mathrm{HC}, 19 \mathrm{DLB}$, and $18 \mathrm{AD}$ patients. Statistical analysis of the motion parameters for the remaining participants revealed no significant differences (Kruskal-Wallis test: translation $p$-value $=0.829, \chi^{2}=0.375, \mathrm{df}=$ 2 ; rotation $p$-value $=0.615, \chi^{2}=0.971, \mathrm{df}=2$ ).

\section{Demographics}

Analysis of the demographic variables is shown in Table 1. Groups were matched by age, gender, and years of education (see Table S4), with DLB and AD patients having similar levels of cognitive impairment (MMSE and CAMCOG). As expected, the three core symptoms of DLB complex visual hallucinations (NPI ${ }^{\text {hall }}$ ), cognitive fluctuations (CAF), and Parkinsonism (UPDRS) were greater in DLB as compared to AD.

\section{Regional homogeneity}

Within each group, the average ReHo maps are shown in Figure 1. In the three groups, high ReHo values were found in precuneus, posterior cingulate and frontal lobes in a cortical pattern that resembled the DMN. Results from between group comparisons are shown in Figure 2 and Table S1. When comparing DLB and HC groups, lower ReHo values were found in sensory-motor areas for DLB, specifically in the left/right pre-central gyri. Higher ReHo (DLB > HC) values were found mainly in the left middle temporal gyrus and right central opercular cortex.

When comparing $\mathrm{AD}$ against $\mathrm{HC}$, lower ReHo values were found in cerebellar regions while higher values were observed in the left/right lingual gyri, left inferior frontal gyrus, and right occipital fusiform gyrus in $\mathrm{AD}$ patients.

Comparisons between patient groups revealed more regions with lower ReHo values in DLB than in $\mathrm{AD}$, including the cerebellum, right precuneus, left lingual, and left post-central gyri. The DLB group also demonstrated higher $\mathrm{ReHo}$ values than the $\mathrm{AD}$ group in the left middle temporal and left temporal fusiform cortices. See Table S1 for cluster details; size, regions, and MNI coordinates. 
Table 1. Demographic, clinical, and cognitive measures

\begin{tabular}{|c|c|c|c|c|}
\hline AND COGNITIVE MEASURES & $\operatorname{DLB}(\mathrm{N}=19)$ & $\mathrm{AD}(\mathrm{N}=18)$ & $\mathrm{HC}(\mathrm{N}=16)$ & $P$-VALUE \\
\hline Male: female & $13: 6$ & $15: 3$ & $13: 3$ & $X_{2}=1.37, p=0.504$ \\
\hline Age; range & $76.32 \pm 6.45 ; 62-89$ & $75.39 \pm 8.6 ; 62-88$ & $76.75 \pm 5.93 ; 62-84$ & $\mathrm{~F}_{2,50}=0.16, p=0.849 \dagger$ \\
\hline MMSE & $23.05 \pm 4.13$ & $21.83 \pm 3.8$ & $29.1 \pm 0.88$ & $t_{35}=0.93, p=0.36^{*}$ \\
\hline UPDRS & $14.95 \pm 5.47$ & $1.56 \pm 1.68$ & $1.44 \pm 1.93$ & $t_{32}=0.19, p=0.851$ \\
\hline CAMCOG & $74.26 \pm 15.2$ & $70.7 \pm 14.5$ & $96.8 \pm 3.03$ & $t_{35}=0.72, p=0.47^{*}$ \\
\hline CAF scale & $4.32 \pm 4.43$ & $0.65 \pm 1.69^{b}$ & na & $t_{34}=3.2, p=0.003^{*}$ \\
\hline NPI total & $5.39 \pm 4.16^{\mathrm{a}}$ & $3.82 \pm 4.12^{\mathrm{b}}$ & na & $t_{33}=1.11, p=0.272^{*}$ \\
\hline NPI hallucinations & $1.53 \pm 1.77$ & $0.0 \pm 0.0^{\mathrm{b}}$ & na & $t_{34}=3.54, p=0.001^{*}$ \\
\hline Years since diagnosis & $1.0 \pm 0.6$ & $1.65 \pm 0.8$ & na & $p$-value $=0.0131$ \\
\hline AChEI (yes:no) & $18: 1$ & 18:0 & na & \\
\hline Levodopa (yes:no) & $8: 11$ & $0: 18$ & na & \\
\hline
\end{tabular}

Values expressed as mean $\pm 1 \mathrm{SD}$.

a $(n=18)$.

$\mathrm{b}(n=17)$.

$\dagger$ ANOVA DLB, AD, and HC.

* Student's $t$-test AD and DLB; 1 Student's $t$-test HC and AD, ttwo-tailed Mann-Whitney $U$ test.

Abbreviations: DLB, dementia with Lewy bodies; AD, Alzheimer's disease; HC, Healthy controls; MMSE, Mini-Mental State examination; CAMCOG, Cambridge Cognitive Examination; NPI, Neuropsychiatric Inventory; CAF, Clinical Assessment of Fluctuating Confusion; VP, Visual Perception; UPDRS, Unified Parkinson's Disease Rating Scale; AChEI, patients on AChEI treatment; Levodopa, patients on Levodopa; na, not applicable.

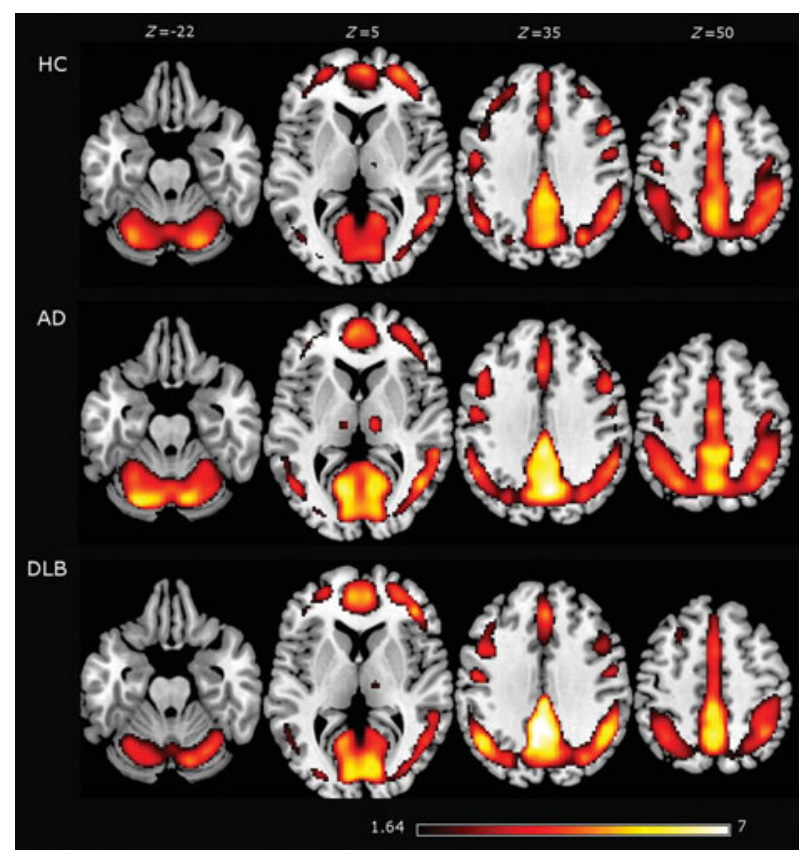

Figure 1. The average regional homogeneity (ReHo) from healthy controls (HCs), Alzheimer's disease (AD), and dementia with Lewy body (DLB) patients. The highest ReHo values were found in the precuneus and frontal cortices in the three groups. Average images are shown with a $t$-score of $>1.64$, which is the threshold for the $\mathrm{HC}$ group when corrected for multiple comparisons ( $p$-value of $<0.05$ corrected). Brain slices are shown in MNI standard space and in neurological convention (the left hemisphere is shown at the left).

Group comparison results derived from anatomical seeds are shown in Figure 3. The strongest difference was found in the left and right post- central gyri ( $p$-value $=0.00078$ and $p$-value $=0.048$, respectively) for $\mathrm{HC}>$ DLB. Other cortical and subcortical regions did not significantly differentiate DLB from HC. When comparing patient groups (DLB vs. AD), the left post-central gyrus and left thalamus showed significantly lower ReHo values in DLB than in AD ( $p$-value $=0.0076$ and $p$-value $=0.026$, respectively).

\section{Correlations with clinical variables}

Correlations between functional seeds and clinical variables were not significant after corrections for multiple comparisons. These results are shown in Table S2. From the anatomical seeds, strong correlations in DLB were observed between the left putamen and UPDRS $(\rho=+0.747, p$-value $<0.001)$ and the left thalamus and MMSE $(\rho=+0.635, p$-value $=0.003)$. Results from the anatomical seeds are shown in Table S3.

\section{Differences in grey matter densities}

The ANCOVA analysis for grey matter volumes in our three groups revealed significant differences in the left hippocampus ( $p$-value $<0.05$, FWE corrected). Post-hoc tests showed that this difference was driven by the $\mathrm{AD}$ group, while the grey matter volume loss in DLB was relatively small, with only a small cluster (two voxels) confined to the right parietal cortex as compared to HC (supplementary material). 


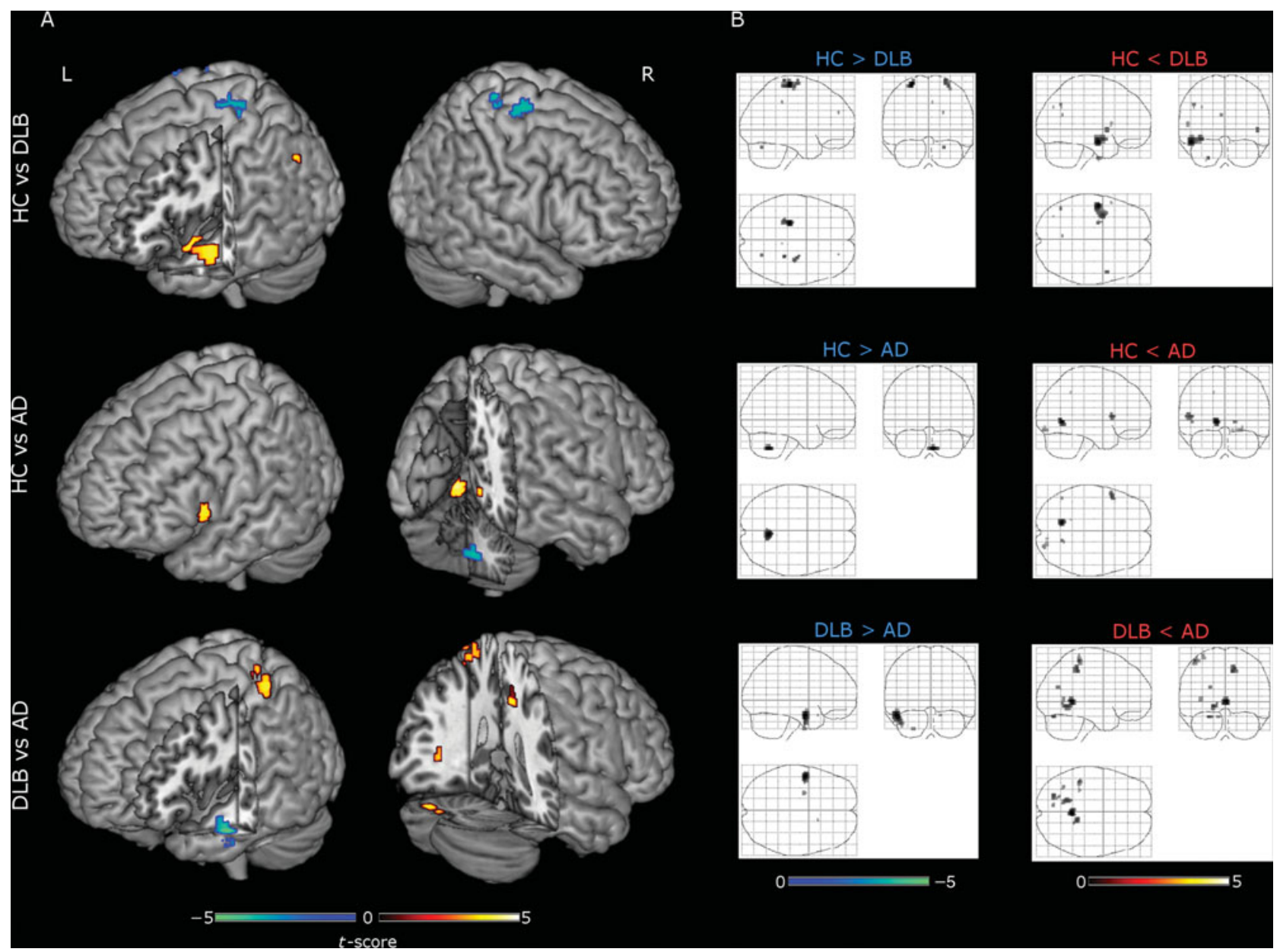

Figure 2. Regional homogeneity (ReHo) group comparisons. Each row shows results in 3D renderings ( $A$ ) and SPM glass brains (B), respectively. First row: healthy controls $(\mathrm{HCs})$ versus dementia with Lewy body (DLB) patients $(\mathrm{HC}>\mathrm{DLB}$ cool colors, $\mathrm{HC}<\mathrm{DLB}$ warm colors). Second row: $H C$ versus Alzheimer's disease $(A D)$ patients ( $H C>A D$ cool colors, $H C<A D$ warm colors). Third row: $D L B$ versus $A D$ (DLB $>A D$ cool colors, DLB $<A D$ warm colors). Results shown at voxel $p$-value $<0.001$ uncorrected with non-parametric permutations $(10,000)$ and presented in neurological convention. A complete list of clusters, regions, and MNI coordinates is given in Table S1.

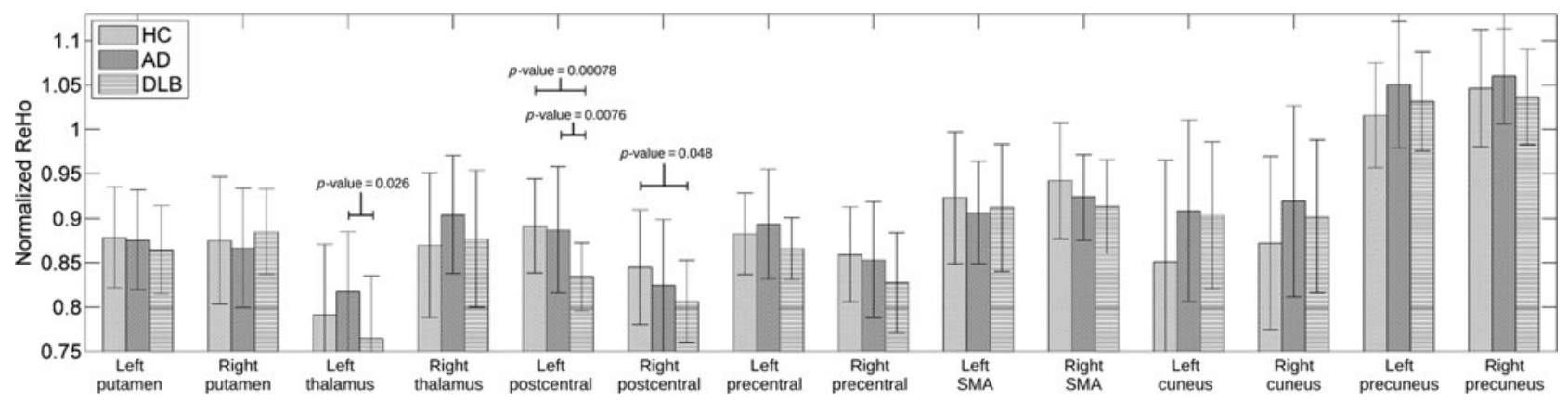

Figure 3. Anatomical seed comparisons of ReHo values. Seeds were defined by the AAL (Anatomical Automatic Labeling) atlas and average normalized ReHo values were estimated from each of the AAL regions using SPM MarsBaR. Right and left post-central gyrus showed significant differences when comparing DLB versus HC.

\section{Discussion}

Our ReHo analysis in DLB and AD patients revealed higher and lower values at several cortical regions. For the DLB group, the lower ReHo values were found mainly in the sensory-motor cortices, while higher ReHo values were found in the left temporal lobe when compared to $\mathrm{HC}$. In $\mathrm{AD}$ patients, higher ReHo values were found in the lingual gyri and lower ReHo values were 
found in the cerebellum, as compared to controls. Differences between patient groups were observed at regions related to both pathologies, e.g. lower $\mathrm{ReHo}$ in temporal cortices in $\mathrm{AD}$ and in posterior brain regions in DLB which mainly encompassed parietal and occipital cortices and might reflect aetiological differences between the diseases.

\section{ReHo differences in AD as compared to HC}

Contrary to previous reports, we were not able to find significant ReHo differences in the precuneus and posterior cingulate cortices in $\mathrm{AD}$ as compared to HC. A post-hoc analysis on these regions revealed that in our $\mathrm{AD}$ group, the average $\mathrm{ReHo}$ value in the precuneus was higher in AD patients than in $\mathrm{HCs}$ and was negatively correlated with the MMSE (see Table S2 and Figure 3). This result disagrees with Zhang et al. (2012) and He et al. (2007), who reported decreased ReHo values in the precuneus and a positive correlation with MMSE in their AD group. An explanation for this discrepancy might be that, in contrast to previous investigations ( $\mathrm{He}$ et al., 2007; Zhang et al., 2012), our AD participants were all taking AChEIs at the time of scanning. AChEI can increase regional cerebral blood flow $(\mathrm{rCBF})$ and partially restore functional connectivity between the posterior cingulate and frontal regions in $\mathrm{AD}$ patients ( $\mathrm{Li}$ et al., 2012).

Another possibility is the compensatory mechanisms reported in neurodegenerative diseases (Grady et al., 2003). In this regard, brain structures that are not yet altered at early disease stages tend to show higher functional activity as an effect of disconnection from those regions that are already pathologically affected (He et al., 2007; Agosta et al., 2012; Damoiseaux et al., 2012). For example, in a longitudinal study in $\mathrm{AD}$ patients, Damoiseaux et al. (2012) reported an increased resting state connectivity in the precuneus when assessing the ventral DMN; however, the authors reported that at later stages, this precuneal hyperconnectivity deteriorated at lower levels than HC (Damoiseaux et al., 2012). The anticorrelation between precuneal ReHo and the MMSE score in our AD participants supports the hypothesis that higher ReHo might be associated with a compensatory response at early disease stages.

In our $\mathrm{AD}$ participants, we found lower ReHo values in the cerebellum as compared to HC. The cerebellum is commonly neglected as a focus of study as it is commonly thought to be a region relatively unaffected by this disease, and previous ReHo investigations in $\mathrm{AD}$ have not reported alterations within this structure. However, the cerebellum is not just implicated in motor control but also appears to play a significant role in cognition, behavior, and attention (Gottwald et al., 2003) - all domains which can be affected in AD. In support of this, He et al. (2007) reported significant positive correlations between cerebellar ReHo values and MMSE in AD, although no significant $\mathrm{ReHo}$ differences were found in this region when compared to HC. Additionally, previous reports showed that cerebellar grey matter loss occurs in AD (Colloby et al., 2014), suggesting an effect caused by the disease process itself.

Higher ReHo values in $\mathrm{AD}$ as compared to the control group were found in several brain regions, mainly the left/right lingual gyri, inferior frontal gyrus, and right occipital fusiform gyrus. He et al. (2007) and Bai et al. (2008) also reported higher ReHo values in these regions in $\mathrm{AD}$, and our results of higher ReHo values also agree with previous investigations reporting compensatory frontal and occipital networks in this disease (Grady et al., 2003; Zhang et al., 2009; Agosta et al., 2012; Damoiseaux et al., 2012).

\section{Higher and lower ReHo values in DLB}

One of the main findings in the current investigation was the low ReHo values in sensory-motor cortices in DLB. The sensory-motor cortices, together with the supplementary motor area, thalamus and putamen, comprise the sensory-motor and basal ganglia resting state networks (Robinson et al., 2009), which are driven by basal thalamocortical connections. Previous research has reported alterations in these networks in PD (Hacker et al., 2012), and our results also concur with previous ReHo investigations reporting lower $\mathrm{ReHo}$ values in sensory-motor areas (Choe et al., 2013). Additionally, Wu et al. (2009a) reported lower ReHo values in the bilateral putamen, supplementary motor areas, and left thalamus. Overall, our findings - in concordance with those seen in PD - reinforce the concept that motorrelated ReHo alterations occur across the Lewy body disease spectrum.

Higher ReHo values were also observed in our DLB group, primarily in the left middle temporal gyrus. Temporal lobe alterations are commonly reported in DLB, and Lewy bodies frequently occur in temporal cortices; however, at the early disease stage, these structures are relatively spared (Watson et al., 2012). Speculatively, higher ReHo values in temporal cortices in DLB may, similar to $\mathrm{AD}$ (Zhang et al., 2009; Agosta et al., 2012; Damoiseaux et al., 2012), reflect a compensatory effect of disease progression. Galvin et al. (2011) reported disconnections between a precuneal seed and temporal cortices in DLB; similarly, Peraza et al. (2014) revealed disconnections between the 
temporal resting state network and the precuneal and posterior cingulate cortex in DLB patients. These results support the idea of local hyperconnectivity within disease-spared regions, triggered by long distance disconnections from disease-affected regions. Contrary to our results, a recent report by Borroni et al. (2015) showed lower ReHo values in DLB compared to HC in the left fusiform gyrus and the pons. We also found a small voxel in the left temporal fusiform gyrus (see Table S2) but showing higher ReHo in DLB compared to HCs, which again might be an effect of disease stages; hyperconnectivity at early stages and hypoconnectivity at later stages. Our participants were recently diagnosed with DLB (average of one year since diagnosed) while participants in Borroni et al. (2015) were on average 4.2 years with the disease. More research will be needed to assess longitudinally the effects of DLB in the functional brain.

\section{ReHo differences between DLB and AD}

When comparing patient groups, ReHo differences were revealed at regions related to both pathologies: DMN and sensory-motor network. The DLB group showed higher ReHo in the left temporal cortex compared with ADs. This result derived from the fact that our DLB group showed higher ReHo in this region than HCs and this difference persisted when compared with the $\mathrm{AD}$ group. A similar reasoning can be applied to the sensory-motor clusters (left post-central gyrus) where the DLB group showed lower ReHo values than AD participants. These results agree with a recent report by our group (Peraza et al., 2015) where we compared the node degree between both patient groups showing lower node degree in temporal cortices in $\mathrm{AD}$ and higher node degree in parietal and occipital cortices in DLB, and which might be related to aetiological divergences between the dieases, e.g. amyloid and synuclein pathologies (Schulz-Schaeffer, 2010).

Limitations in our study are the relatively small significant differences observed between groups, and this could be due to the mild disease stage in our patients. Motion artifacts can also be an issue for ReHo; however, we minimized these artifactual sources with a strict motion exclusion criteria and regression of motion parameters. Furthermore, we also checked that the motion levels in our three groups were not significantly different (i.e. patient groups did not move significantly more than controls during the MRI scanning). Another factor that might influence ReHo is grey matter loss (He et al., 2007), however, previous investigations have reported that grey matter loss tends to slightly affect cluster sizes with relatively constant results (Damoiseaux et al., 2012). In our participants, such grey matter losses were not widespread and they were in regions not cortically overlapped with ReHo clusters; therefore, we would argue that our results in local synchronizations are not heavily influenced by grey matter differences.

Another factor in the present study was the potential effect of cholinergic and dopaminergic medications in our DLB patients (18 patients on AChEIs and eight patients on Levodopa after movement exclusion criteria). Previous rs-fMRI investigations assessing AChEI treatment effects in $\mathrm{AD}$ (Li et al., 2012) and evaluating the effects of Levodopa on ReHo values in PD (Choe et al., 2013) reported that these medications were able to restore or normalize the levels of local and global synchronizations. Hence, medications in $\mathrm{AD}$ and DLB appear to restore ReHo measures to control levels, but are unlikely to cause higher or lower ReHo values than in HC. This supports the notion that the higher ReHo values in our results are more likely to be compensatory responses at early disease stages. However, one rs-fMRI investigation in PD assessing Levodopa treatment and its effects in node degree - a measure similar to ReHo but also influenced by long distance synchronizations - reported changes from lower (in the "OFF" medication state) to higher (in the "ON" state) node degree in the cerebellar, basal regions, and parietal cortices as compared to HC (Wu et al., 2009b). Effects of other medications such as antidepressants should also be considered, however only a small fraction of our study group were taking antidepressants at the time of scanning; $1 \mathrm{HC}, 2 \mathrm{AD}$, and $2 \mathrm{DLB}$ participants. We are also cautious in over-interpreting the correlations between clinical variables and ReHo values, given the uncorrected nature of the analyses. However, these suggestive associations may foster further investigations, for example, in understanding the role of motor networks in cognitive dysfunction in DLB.

\section{Conflict of interest}

None.

\section{Description of author's roles}

L.R. Peraza designed the imaging analysis, statistics, and wrote the first manuscript draft. S.J. Colloby performed the anatomical seed analysis and revised the manuscript. L. Deboys contributed in the imaging analysis. J.T. O'Brien reviewed the manuscript. M. Kaiser reviewed the manuscript. J.$\mathrm{P}$ Taylor is principal investigator, oversaw the study, and revised the manuscript. 


\section{Acknowledgments}

This work was supported by an Intermediate Clinical Fellowship (WT088441MA) to J-P.T., the National Institute for Health Research (NIHR), Newcastle Biomedical Research Unit (BRU) based at Newcastle upon Tyne Hospitals NHS Foundation Trust, and Newcastle University.

\section{Supplementary material}

To view supplementary material for this article, please visit http://dx.doi.org/10.1017/ S1041610216000429.

\section{References}

Agosta, F., Pievani, M., Geroldi, C., Copetti, M., Frisoni, G. B. and Filippi, M. (2012). Resting state fMRI in Alzheimer's disease: beyond the default mode network. Neurobiology of Aging, 33, 1564-1578.

Andrews-Hanna, J. R., Reidler, J. S., Huang, C. and Buckner, R. L. (2010). Evidence for the default network's role in spontaneous cognition. Fournal of Neurophysiology, 104, 322-335.

Bai, F. et al. (2008). Default-mode network activity distinguishes amnestic type mild cognitive impairment from healthy aging: a combined structural and resting-state functional MRI study. Neuroscience Letters, 438, 111-115.

Borroni, B. et al. (2015). Structural and functional imaging study in dementia with Lewy bodies and Parkinson's disease dementia. Parkinsonism E Related Disorders, 21, 1049-1055.

Brett, M., Anton, J. L., Valabregue, R. and Poline, J. B. (2002). Region of interest analysis using an SPM toolbox. (Abstract 10511. In Presented at the 8th international conference on functional mapping of the human brain, June 2-6, 2002, Sendai/Japan) [available on CD-ROM]. NeuroImage, 16.

Choe, I. H., Yeo, S., Chung, K. C., Kim, S. H. and Lim, S. (2013). Decreased and increased cerebral regional homogeneity in early Parkinson's disease. Brain Research, 1527, 230-237.

Colloby, S. J., O'Brien, J. T. and Taylor, J. P. (2014). Patterns of cerebellar volume loss in dementia with Lewy bodies and Alzheimers disease: a VBM-DARTEL study. Psychiatry Research, 223, 187-191.

Damoiseaux, J. S., Prater, K. E., Miller, B. L. and Greicius, M. D. (2012). Functional connectivity tracks clinical deterioration in Alzheimer's disease. Neurobiology of Aging, 33, e819-830.

Donaghy, P., Thomas, A. J. and O'Brien, J. T. (2015). Amyloid PET imaging in Lewy body disorders. The American fournal of Geriatric Psychiatry, 23, 23-37.

Galvin, J. E., Price, J. L., Yan, Z., Morris, J. C. and Sheline, Y. I. (2011). Resting bold fMRI differentiates dementia with Lewy bodies versus Alzheimer disease. Neurology, 76, 1797-1803.
Gottwald, B., Mihajlovic, Z., Wilde, B. and Mehdorn, H. M. (2003). Does the cerebellum contribute to specific aspects of attention? Neuropsychologia, 41, 14521460.

Grady, C. L., McIntosh, A. R., Beig, S., Keightley, M. L., Burian, H. and Black, S. E. (2003). Evidence from functional neuroimaging of a compensatory prefrontal network in Alzheimer's disease. The fournal of Neuroscience, 23, 986-993.

Hacker, C. D., Perlmutter, J. S., Criswell, S. R., Ances, B. M. and Snyder, A. Z. (2012). Resting state functional connectivity of the striatum in Parkinson's disease. Brain, 135, 3699-3711.

He, Y. et al. (2007). Regional coherence changes in the early stages of Alzheimer's disease: a combined structural and resting-state functional MRI study. NeuroImage, 35, $488-500$.

Li, W. et al. (2012). Changes in regional cerebral blood flow and functional connectivity in the cholinergic pathway associated with cognitive performance in subjects with mild Alzheimer's disease after 12-week donepezil treatment. NeuroImage, 60, 1083-1091.

McKeith, I. G. et al. (2005). Diagnosis and management of dementia with Lewy bodies: third report of the DLB consortium. Neurology, 65, 1863-1872.

McKeith, I. et al. (2007). Sensitivity and specificity of dopamine transporter imaging with 123I-FP-CIT SPECT in dementia with Lewy bodies: a phase III, multicentre study. Lancet Neurology, 6, 305-313.

Mukaetova-Ladinska, E. B. (2015). Molecular imaging biomarkers for dementia with Lewy bodies: an update. International Psychogeriatrics, 27, 555-577.

Peraza, L. R. et al. (2014). fMRI resting state networks and their association with cognitive fluctuations in dementia with Lewy bodies. NeuroImage: Clinical, 4, 558565.

Peraza, L. R., Taylor, J. P. and Kaiser, M. (2015). Divergent brain functional network alterations in dementia with Lewy bodies and Alzheimer's disease. Neurobiology of Aging, 36, 2458-2467.

Robinson, S. et al. (2009). A resting state network in the motor control circuit of the basal ganglia. $B M C$ Neuroscience, 10, 137.

Schulz-Schaeffer, W. J. (2010). The synaptic pathology of alpha-synuclein aggregation in dementia with Lewy bodies, Parkinson's disease and Parkinson's disease dementia. Acta Neuropathologica, 120, 131-143.

Song, X.-W. et al. (2011). REST: a toolkit for resting-state functional magnetic resonance imaging data processing. PLoS ONE, 6, e25031.

Watson, R., O'Brien, J. T., Barber, R. and Blamire, A. M. (2012). Patterns of gray matter atrophy in dementia with Lewy bodies: a voxel-based morphometry study. International Psychogeriatrics, 24, 532-540.

Wu, T. et al. (2009a). Regional homogeneity changes in patients with Parkinson's disease. Human Brain Mapping, 30, 1502-1510.

Wu, T., Wang, L., Chen, Y., Zhao, C., Li, K. and Chan, P. (2009b). Changes of functional connectivity of the motor network in the resting state in Parkinson's disease. Neuroscience Letters, 460, 6-10. 
Wu, T., Zang, Y., Wang, L., Long, X., Li, K. and Chan, P. (2007). Normal aging decreases regional homogeneity of the motor areas in the resting state. Neuroscience Letters, 423, 189-193.

Zang, Y., Jiang, T., Lu, Y., He, Y. and Tian, L. (2004). Regional homogeneity approach to fMRI data analysis. NeuroImage, 22, 394-400.
Zhang, H. Y. et al. (2009). Detection of PCC functional connectivity characteristics in resting-state fMRI in mild Alzheimer's disease. Behavioural Brain Research, 197, 103-108.

Zhang, Z. et al. (2012). Altered spontaneous activity in Alzheimer's disease and mild cognitive impairment revealed by regional homogeneity. NeuroImage, 59, 1429-1440. 